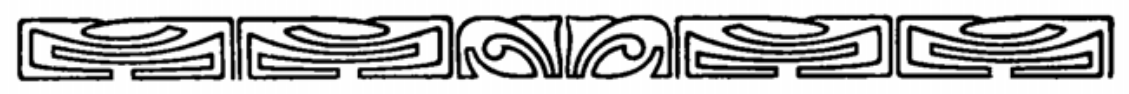

\title{
Vorwort zur ersten Auflage
}

Wenn im Laufe der Erdgeschichte irgendein Landtier tot zu Boden sank und dann von der Natur mit Hilfe des Wassers eingespült wurde in ein Wasserbecken, auf dessen Boden es in die sich bildenden Sedimente eingebettet ward, dann liegt es begraben in Schichten desselben geologischen Zeitalters, während dessen es gelebt hatte. Aus dem Alter dieser Schichten können wir daher stets mit Sicherheit, falls nicht etwa ausnahmsweise eine Umlagerung stattfand, auf das geologische Alter dieses Landtieres schließen.

Anders beim Menschen. Zwar kommt es natürlich auch hier vor, daß er durch die Natur nach seinem Tode in Sedimente eingehüllt wird, wie jene Tiere, so daß er dann das geologische Alter jener Sedimente hat. Aber sehr häufig oder meistens ward dem Menschen nicht durch die Natur, sondern durch Menschen die letzte Ruhestätte bereitet; und dann ward er begraben in Schichten, welche zufällig dort anstanden und bereits einer vergangenen, vielleicht längst dahingegangenen Zeit angehörten. Er hat daher, wenn man später seine Knochen findet, scheinbar das hohe Alter dieser Schichten und ist doch in Wirklichkeit sehr viel jünger als diese. Aus dem Alter von Schichten, in denen Menschenknochen liegen, können wir daher keineswegs immer mit Sicherheit auf das geologische Alter des Menschen schließen; wir können vielnuehr in dieser Beziehung $\mathrm{zu}$ argen Fehlschlüssen verleitet werden, falls nicht mit ins Grab gegebene letzte Geschenke oder andere Umstände uns anzeigen, daß es sich hier um eine Begräbnisstätte handelt.

Die nicht genügende Beachtung dieser Verhältnisse, vereint mit der nicht genügenden Sorgfalt, mit welcher man die Lagerungsverhältnisse feststellte, haben dahin geführt, daß die Angabell über das geologische Alter fossiler Menschenreste, die in früheren Zeiten gefunden wurden, vielfach ganz unsicher sind und ewig unsicher bleiben werden. Das ist der Grund, warum ich, um nicht Unsicheres fälschlich als sicher hinzustellen, im Jahre 1901 nicht 
von dem ,diluvialen", sondern von dem, ,alten" Menschen sprach; indem ich darunter verstand die sehr wenigen zweifellos diluvialen und die viel zahlreicheren fraglich diluvialen, zum Teil wohl auch alluvialen Menschenreste, die man damals kannte. ${ }^{1}$

Inzwischen ist fast ein Jahrzehnt vergangen und in dieser kurzen Frist haben sich so viel neue und bezüglich ihres geologischen Alters sichergestellte Funde von fossilen Menschenresten ergeben, daß ich im folgenden stets von dem, ,diluvialen“ Menschen reden kann. Es liegt auf der Hand, daß, wenn diese Erfunde im selben Maße fortschreiten, wir binnen einem halben Jahrhundert ein ganzes kleines Museum aus den vereinigten Resten des fossilen Menschen werden zusammenstellen können. Unsere Kenntnis des letzteren wird dann eine sehr viel gründlichere geworden sein. Aber im großen und ganzen werden dann - so will mir scheinen - unsere heute bereits erlangten Kenntnisse der beiden Schädeltypen des diluvialen Menschen, wenn auch erweitert, so doch als in ihrer jetzigen Grundlage zu Recht bestehend ihre Gültigkeit behalten.

Im Jahre 1901 hatte ich dem fünften internationalen Zoologenkongre $\beta$ in Berlin über den damaligen Stand unserer Kenntnisse bezüglich des fossilen Menschen zu berichten. ${ }^{2} \mathrm{DaB}$ der diluviale Mensch, zunächst einmal in tertiärer Zeit, Vorfahren gehabt haben müsse, war für Zoologen ohne weiteres selbstverständlich. Es konnte sich daher nicht darum handeln, dem Kongreß irgendwelche Spekulationen vorzutragen, wie dieser tertiäre Mensch ausgesehen haben und aus weichen Vorfahren er hervorgegangen sein könnte. Das aber um so weniger, als wir Sicheres darüber nicht wissen.

Ich habe daher damals das zusammengefaßt, was sich an positiven paläontologischen Daten des diluvialen und an negativen des tertiären Menschen mit Sicherheit aussagen ließ. Von diesem Sicheren habe ich getrennt das Spekulative, speziell das, was sich aus den damals neuen Untersuchungen Friedenthals bezüglich der Blutsverwandtschaft von Menschen und Menschenaffen ergab; Untersuchungen, aus welchen ein recht enger Grad von Blutsverwandtschaft beider hervorzugehen scheint.

${ }^{1}$ Das ist ein Umstand, dem P. Erich Wasmann in seiner Inhaltsangabe meines Vortrages nicht geniigend Rechnung getragen hat.

${ }^{2}$ W. Branca (Branco), Der fossile Mensch. Verhandlungen des fünften internat. Zoologenkongresses zu Berlin 1901. Jena bei Gustav Fischer 1902. Sonderabdruck S. $1-25$ (5 Textfiguren). 
Der damalige (1901) Stand unserer paläontologischen Kenntnisse vom Menschen läßt sich dahin zusammenfassen:

A. 1. In "alter" Zeit haben wir zwei Typen von Menschenschädeln: Der eine häufigere, schon ganz wie der unsere, also lıöher stehend. Der andere, damals noch seltene, mit den bekannten Eigenschaften des Neandertaler Schädels, also von niedererer Bildung.

2. Die bisherigen Angaben über Menschenknochen zweifellos tertiären Alters sind sämtlich mehr oder weniger fragwürdig.

3. Das pliocäne Alter des Pithecanthropus, dessen Schädel sich eng an jenen inferioren Typus des diluvialen Menschen anschließt, ist so fraglich, daß wir von ihm als einem angeblich sicheren tertiären Vorfahren des Menschen vorerst noch ganz absehen müssen.

Seitdem ist bald ein Dezennium verflossen; und entsprechend dem allgemeinen Fortschritte unserer paläontologischen und geologischen Kenntnisse sind auch speziell die bezüglich des fossilen Menschen vorangeschritten. Aber das Ergebnis ist doch im großen und ganzen noch dasselbe geblieben wie 1901.

Punkt 1 (zwei Typen von Schädeln) hat sich jetzt dahin verschoben:

a) daß der diluviale inferiore Neandertal-Typus nicht nur an weiteren Stellen im Westen Europas, sondern auch im Süden und im Osten entdeckt worden ist; so daß nun eine ehemals größere Verbreitung desselben sich ergibt, als das 1901 der Fall war.

b) daß aber das diluviale Alter des Neandertal-Schädels ${ }^{1}$ durch Rauffs Untersuchungen nun endgültig als absolut zweifelhaft erwiesen worden ist. Der Neandertaler Schädel muß endlich aus der Reihe der angeblich sicher diluvialen Schädel ausscheiden; wenngleich man natürlich nach wie vor von dem Neandertaler Schädeltypus sprechen kann und ich das auch hier tue.

c) daß dieser diluviale inferiore Schädeltypus auch heute noch lebend unter Australiern gefunden worden ist, wodurch die Abzweigung dieses diluvialen Neandertal-Typus von Homo sapiens als einer besonderen Menschenart, wie sie von Wilser und Schwalbe seinerzeit befürwortet wurde und werden konnte, jetzt nicht mehr

' Welches Alter, weil der Schädel von Arbeitern ohne jede Feststellung der Lagerung in einer Höhle gefunden worden war, den mit dieser geologen Frage sich beschäftigenden Geologen schon längst unsicher erschienen ist; was übrigens auch Virchow stets behauptet hat. 
haltbar ist; dies aber um so weniger, als $\mathrm{H}$. Klaatsch sogar von dem Australierschädel berichtet, daß er noch inferiorer als der Neandertaler ist. Solange man also den lebenden Menschen nicht in verschiedene Spezies teilt - was man wohl mit Recht tun könnte -solange wird man auch den Neandertaler Typus nicht als besondere Art betrachten dürfen.

Immerhin ist die Frage, ob besondere Art oder nicht, doch eine mehr formale; das Tatsächliche bleibt bestehen, das Schwalbe durch seine ausgezeichnet scharfen Untersuchungen für diesen Typus festgestellt hat.

d) daß aber ebenso auch von den höher organisierten diluvialen Menschenschädeln seitdem neue Erfunde gemacht worden sind, so daß sich also auch deren ehemalige Verbreitung als eine größere erweist.

e) daß dieser diluviale höhere Schädeltypus möglicherweise sogar ebenso alt oder noch älter in Europa sein kann, als jener inferiore (Schädel von Galley-Hill).

B. Punkt 2 hat sich dagegen für Europa noch gar nicht verschoben. Es bleibt sicher, daß wir Menschenknochen tertiären Alters hier bisher noch nicht gefunden haben. Auch für Nordamerika und Asien gilt das wohl. Zwar ein Menschenzahn, den Frau Selenka von Java mitgebracht hat - er ist nicht bei Trinil, sondern bei Sonde gefunden -, scheint mir sicher fossil zu sein. Ob er aber diluvialen oder tertiären Alters ist, läßt sich nicht sagen.

Kurz vor Drucklegung dieser Arbeit erschienen nun aber noch zwei Arbeiten von Lehmann-Nitsche und Fl. Ameghino; nach beider Ansicht soll der menschliche Halswirbel, ein Atlas, den sie beschreiben, tertiären Alters sein. Zudem aus Südamerika! das wäre das doppelt Auffallende und Interessante.

C. Punkt 3 hat sich dahin verschoben, daß die Zweifel, welche ich im Jahre 1901 an dem pliocänen Alter des Pithecanthropus äußern konnte und mußte, jetzt durch die Untersuchungen mehrerer Geologen, welche an Ort und Stelle die Lagerungsverhältnisse einer genaueren Prüfung unterworfen haben, und durch die Untersuchung mehrerer Paläontologen, welche die in jenen Schichten vorkommenden fossilen Mollusken und Pflanzen untersucht haben, noch viel mehr verstärkt worden sind, als es damals der Fall war.

Vorerst also, bis zu einer endgültigen Entscheidung über das Alter des Pithecanthropus, erscheint noch mehr Vorsicht, als mir 1901 nötig schien, geboten gegenüber solchen 
Spekulationen, welche den Pithecanthropus ohne weiteres als tertiären Ahn in die direkte Ahnenreihe des Menschen stellen wollen.

Aus dieser Zusammenfassung folgt also, daB das, was ich im Jahre 1901 in meinem Vortrag vor dem internationalen Zoologenkongre $B$ in Berlin feststellen konnte, durch heutige Erfahrung meist nur noch verstärkt wird; bei Absehen von der Modifikation, da $B$ besonders die Zahl der diluvialen Schädel des inferioren Typus, aber auch diejenige des hoheren Typus, sich vermehrt hat, so daß jetzt also der niedere Typus sich als in damaliger Zeit verbreiteter gewesen crweist, als aus den damaligen überaus spärlichen Erfunden sicher diluvialer Vorkommen desselben hervorging. Sodann bei Absehell von dem erwähnten Atlas, der tertiären Alters sein soll.

Wenu ich in meinen Ausführungen an gewissen Funden der fossilen Schädel (und Knochen), sowie in bezug auf andere Fragen Kritik zu üben suche, meine abweichende Ansicht ausspreche, so geschieht das wahrlich nicht in dem Bestreben, besser wissen zu wollen; sondern es ist geradezu unvermeidlich für jeden, der es unternimmt, den gegenwärtigen Zustand unserer Kenntnisse von dem wichtigsten Gegenstande der Paläontologie, dem fossilen Menschen, darzulegen.

Als „Schlußbetrachtungen“ angefügt habe ich die Besprechung einer Reihe von Fragen allgemeiner Natur, die nicht speziell den fossilen Menschen, sondern den Menschen überhaupt angehen. Sie beziehen sich auf Entstehung des Lebens, auf mono- oder polyphyletische Herkunft der Lebewelt, kirchliche und naturwissenschaftliche Dogmen, Religion und Monismus.

Berlin, August 1910 .

W. B. 